\title{
Reduced emergency calls and improved weekend discharge after introduction of a new electronic handover system
}

\section{Boloor S Rao \\ MB BS, DNB \\ Registrar \\ Gail O Lowe \\ RN, BN, MHA Project Officer, Department of General Medicine ${ }^{2}$ \\ Andrew J Hughes \\ MB BS, FRACP \\ Director, Department of \\ General Medicine and Service Redesign Unit ${ }^{2}$ \\ 1 General Medicine Flinders Medical Centre Adelaide, SA. \\ 2 Barwon Health Geelong, VIC \\ andrewhu@ barwonhealth.org.au}

MJA 2012; 197: 569-573 doi: 10.5694/mjall.11048 lthough it is widely accepted that good handover is important for patient safety, there is only limited evidence that improved handover can lead to reduced patient risk. $^{1,2}$

Written handover transmits information more reliably than informal communication, especially in conjunction with a verbal component. ${ }^{3}$ Before this initiative, written weekend handover in general medicine at Barwon Health was variable in content and non-standardised, and information was located in a variety of places. Such non-standardised handovers usually only comprised clinical summaries. We felt this was both a risk to our patients and inefficient for the organisation. It was difficult to ensure that key clinical messages were transmitted across multiple shifts of covering doctors. There was constant pressure for patients to be discharged on weekends, and this was difficult to organise unless instructions could be conveyed to the doctors covering the weekend shifts.

We sought to improve our doctorto-doctor weekend shift handovers through a system where handover components could be entered electronically. We were aware of other systems such as SBAR (situationbackground-assessment-recommendation), ISBAR (identify-situation-background-assessmentrequest) and ISoBAR (identify-situation-observations-backgroundagreed plan-read back), but felt these were better for direct person-to-person communication of time-critical information requiring immediate action or escalation. ${ }^{4-6}$ We required a system of communicating written information to multiple staff members, for actioning in the future (over multiple shifts) in a non-urgent and ongoing manner. None of these existing communication systems contained all of the components we required, ${ }^{4,5,7,8}$ so we set out to develop our own.

Objectives: To measure the frequency and content of electronic handover before and after implementation of the Blue BARRWUE handover system, and to measure its effect on patient safety and hospital efficiency over weekends.

Design, setting and participants: Point-prevalence study comparing outcomes for general medical inpatients present over weekends before implementation (1 May 2008 to 30 April 2009) and after implementation (1 May 2009 to 30 April 2010) of the Blue BARRWUE handover system at Geelong Hospital.

Intervention: Implementation of the Blue BARRWUE handover system and its components (updated working diagnosis, background, alerts, resuscitation status, requests, who to do what and when, updates and executable discharge plan).

Main outcome measures: Presence of any written handover notes or updated working diagnoses in the BOSSnet clinical information system, content of handover notes, frequency of weekend discharges and medical emergency team (MET) calls before and after implementation.

Results: In the 12 months before implementation of the Blue BARRWUE handover system, 976 patients (47.98\%) had a handover note in BOSSnet, versus 1646 patients (95.09\%) in the 12 months after implementation $(P<0.001$; rate ratio [RR], 20.75; 95\% Cl, 16.33-26.44). Before implementation, 289 patients (14.21\%) were discharged over weekends, versus 353 patients (20.39\%) after implementation, $(P<0.001$; RR, 1.44; 95\% Cl, 1.25-1.65). MET calls were made for 152 general medical patients before implementation (7.47\%), versus 95 general medical patients (5.49\%) after implementation ( $P=0.01$; RR, 0.73; 95\% Cl, 0.57-0.94).

Conclusions: The Blue BARRWUE system has sustainably improved written handover in our organisation and was associated with improvement in both patient safety and hospital efficiency.

Barwon Health is the largest regional health care provider in Victoria, caring for a catchment of about 500000 people. Its acute site is the Geelong Hospital, which comprises 406 beds, including 69 beds allocated to five general medical units. During the week, each unit is staffed by an intern, a medical registrar and a consultant physician, who participate in a roster to receive all general medical patient admissions and cover each other on weekends. On the weekend, one registrar and one intern cover all new admissions and inpatients for all five units. The average number of general medical patients admitted per day is 11.

Barwon Health is committed to patient safety. A medical emergency team (MET) system was introduced in 2003 to identify patients with a deteriorating condition using standard criteria, and to prevent adverse events. ${ }^{9-11}$ Since 2007, the MET call rate has been stabilising at about 60 calls per 1000 admissions across Barwon Health, which is consistent with the rate of emergency calls expected in a mature established system, ${ }^{12-15}$ although the number of MET calls in general medicine had been increasing up to the time of this study. The intensive care registrar enters the details of all MET calls into SLIC, a locally developed comprehensive clinical information and data collection system for the intensive care unit.

Barwon Health uses the BOSSnet clinical information system (Core Medical Solutions). This allows doctors to create customisable lists of their patients, provides access to pathology and imaging results, and contains an electronic medical record comprising scanned documents from previous admissions, outpatient notes and correspondence.

Useful handover information already in BOSSnet includes: patient name, date of birth, unique identifier, admission date, unit and specialist, 
1 Computer screen image showing the interface for entering the handover information in the BOSSnet clinical handover system (mock patient data)

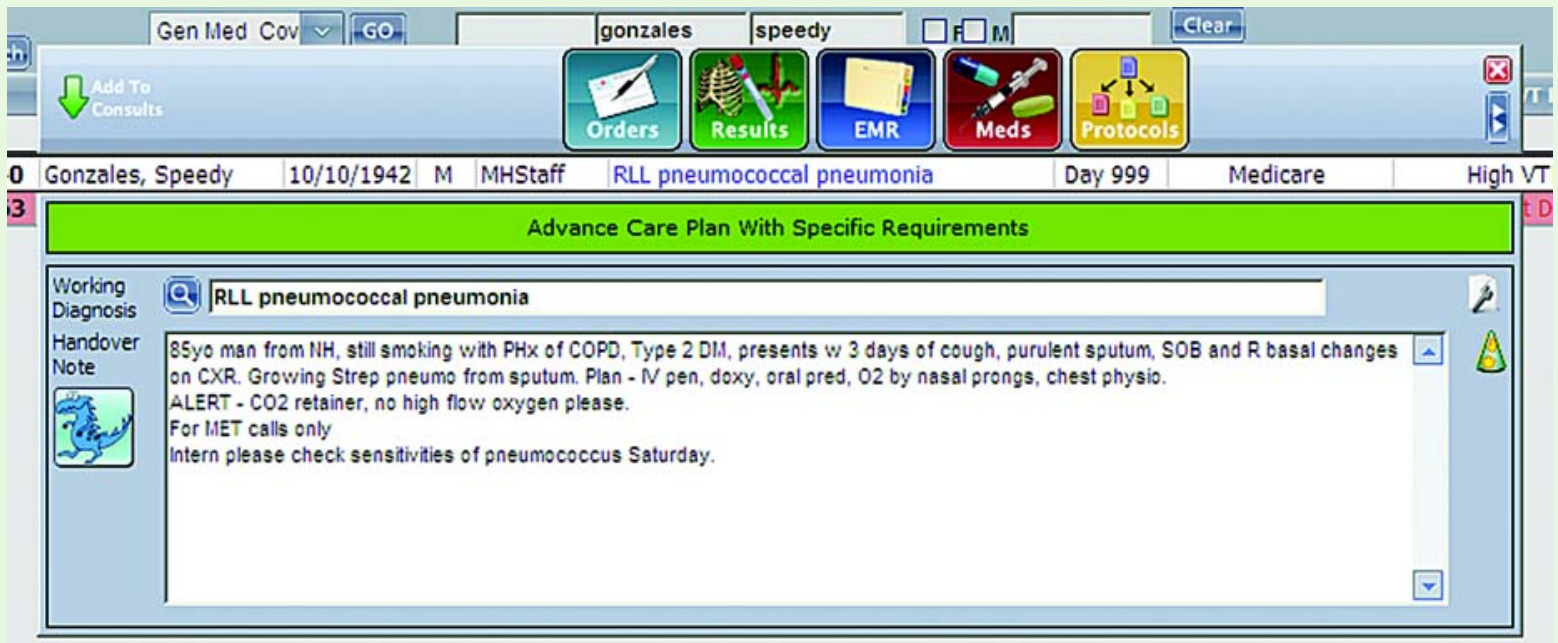

working diagnosis and a free-text box for handover notes.

Covering doctors print patient lists including the above information and recent test results at the start of a shift and access information electronically through the day as required.

This study had two major aims: first, to measure the frequency and content of written electronic handover in BOSSnet on Friday evenings before and after implementation of the new handover system for general medical inpatients; and second, to measure the effect of the new handover system on patient safety and hospital efficiency over weekends.

\section{Methods}

\section{Development and implementation of the handover process}

In April 2009, we held a brainstorming session with around 15 general medical interns, registrars and a physician. The session was focused on the components and order of the handover as well as a discussion on why doctors handed over certain patients and what messages they were trying to convey. The components of the handover were placed in an order that corresponded to the way that the handover was structured by experienced doctors at our institution. The acronym "Blue BARRWUE" was created from the initials of the components and a mascot was developed. Reminders about handover were put in place, including automatic paging on Fridays at $12 \mathrm{pm}$. Combined lists of inpatients belonging to all five general medical units (shared views) were developed to be printed by covering doctors at the beginning of their shift. Teams were encouraged to update their handovers daily as a shared responsibility between intern and registrar (Box 1). This task took about 510 minutes at the end of each day. Particular emphasis was placed on Friday handovers to ensure that plans were in place for the weekend for covering doctors to act on. Electronic reports were developed to monitor completion rates. Posters about the new handover system were displayed on the medical wards. New doctors received training in using the system alongside ISBAR. In addition, nurses on the medical wards began printing and reading the handovers to help them plan for weekends.

\section{The components of the Blue BARRWUE system}

"Blue" updated working diagnosis: When the working diagnosis is updated in BOSSnet to make it accurate, its colour changes to blue. Updating the working diagnosis was considered the first step in a reliable handover. A working diagnosis had always been entered in BOSSnet in the emergency department, but sometimes this was only the preliminary diagnosis.

Background (to the working diagnosis): This includes a summary of the presenting history, social situation, other active problems, examination findings, investigation results, procedures and management plan. In the past, summaries in BOSSnet would often become too long and outdated. However, when performing a verbal handover, experienced doctors would usually edit the outdated information and summarise the patient's background in a couple of lines.

To replicate this, we encouraged the teams to treat the summary like a "haiku" that elegantly summarised the patient in three lines in combination with the updated working diagnosis. We strongly encouraged doctors to delete and update outdated information (which is stored in the background for medicolegal purposes).

Alerts: Conceptually, when a doctor handed over a patient, it was often to alert the covering doctor(s) to something, eg, "UNSTABLE". These alerts are capitalised to make them stand out in the handover note.

Resuscitation status: Covering doctors considered resuscitation status critical, particularly when asked to review patients in an unstable condition.

Requests: These are what the doctor doing the handover is asking the covering doctor to do (eg, check results, monitor fluid status).

Who to do what and when: We felt it was important for interns and registrars to share a single handover to prevent their handovers diverging. Thus, stating "who" is to do the requests and "when" was necessary to divide tasks in a combined handover.

Updates: The handover note is updated over the course of the week- 
2 Proportion of general medical inpatients with a handover note and an updated working diagnosis, and average proportions before implementation of the Blue BARRWUE electronic handover system*

_ Proportion of patients with a handover note

--- Average proportion of patients with a handover note before implementation

- Proportion of patients with an updated working diagnosis

- - Average proportion of patients with an updated working diagnosis before implementation

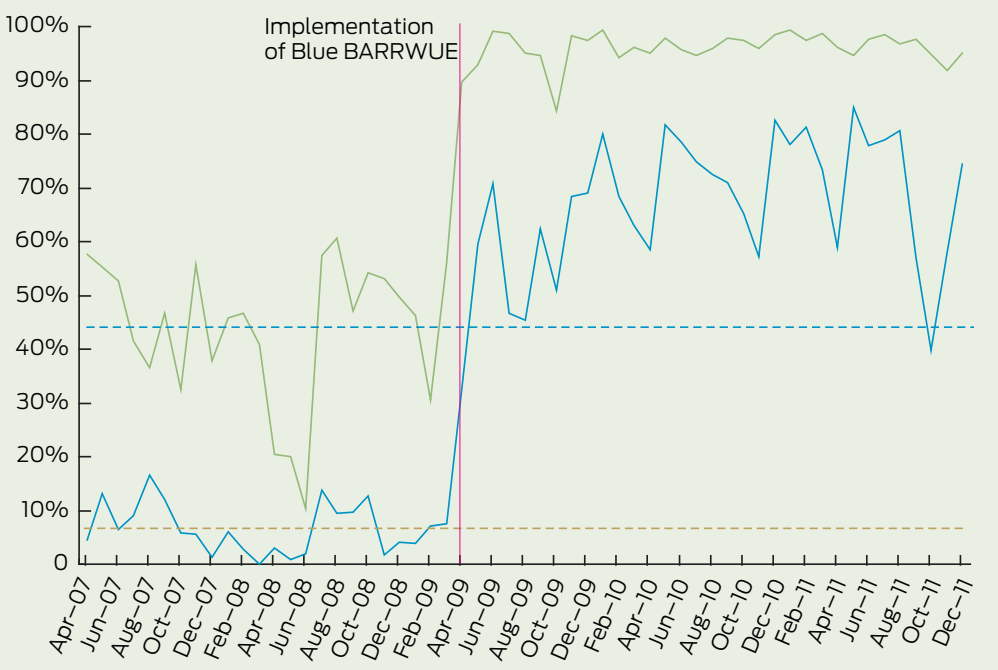

* Proportion of patients with a handover note and proportion of patients with an updated working diagnosis crossed upper control lines after intervention (not shown).

end. This is useful for hand-on to the next shift and hand-back after the weekend.

Executable discharge plan: This includes criteria for discharge, who is to see the patient and discharge him or her, information about the discharge prescription and a follow-up plan.

\section{Audit process}

The study was performed as a quality improvement project and designed and implemented over a short period of time. Because of this, we required measures for which existing data were available.

All general medical inpatients at $7 \mathrm{pm}$ on Friday evenings were included in the audit with the following exceptions: patients who were admitted on the days of the audit (as many of these were still in the process of being admitted at $7 \mathrm{pm}$ and did not have a handover note recorded yet), patients under the care of private consultants and patients in the intensive care unit (as different handover systems were used for these patients).

Working diagnoses and handover notes for patients were extracted from BOSSnet, admission and discharge information was obtained from i.PM (iSOFT) patient management soft- ware, and MET and code blue (cardiac arrest or other life-threatening emergency call) data were taken from SLIC.

We chose to audit weekend discharges as a measure of hospital efficiency, because in our experience, these rely heavily on the provision of a discharge plan in the handover, and failure to provide one often results in patients remaining in hospital unnecessarily over the weekend, which is frustrating for them and inefficient for the hospital.

We chose MET calls as a measure of safety because they often occur for patients who have only gradually become physiologically unstable. We hypothesised that with systematic handover and review of appropriate patients over the weekend, these might occur less frequently.

As part of the audit, we checked for the presence of any handover notes or updated working diagnoses in BOSSnet at $7 \mathrm{pm}$ on Fridays for all general medical inpatients at Geelong Hospital. We then compared the results from the 12 months before implementation (1 May 2008 to 30 April 2009) and the 12 months after implementation (1 May 2009 to 30 April 2010).
We carried out an audit of the content of all general medical inpatient handover notes that were present in BOSSnet at $7 \mathrm{pm}$ on each Friday over 4 consecutive weeks, starting with the first Friday in November 2009, 6 months after implementation of Blue BARRWUE. We also audited the handover notes from the corresponding four Fridays in 2008 and compared the results from the two periods. One of us (AJH) did the audit in a blinded fashion and was not aware if each handover was from before or after implementation of Blue BARRWUE. The audit was based on components of the Blue BARRWUE system.

We also audited weekend discharges over the 12 months before implementation (1 May 2008 to 30 April 2009) and over the 12 months after implementation (1 May 2009 to 30 April 2010).

Finally, we performed an audit of general medical and non-general medical MET calls and code blue calls on weekends over the 12 months before implementation (1 May 2008 to 30 April 2009) and the 12 months after implementation (1 May 2009 to 30 April 2010).

\section{Statistics}

Chi-square analysis or the Fisher exact test was performed on all parametric data, and a $P$ value of 0.05 was considered statistically significant.

\section{Proportion of handover components present in the BOSSnet clinical handover system at $7 \mathrm{pm}$ on Friday evenings in November 2008 and November 2009}

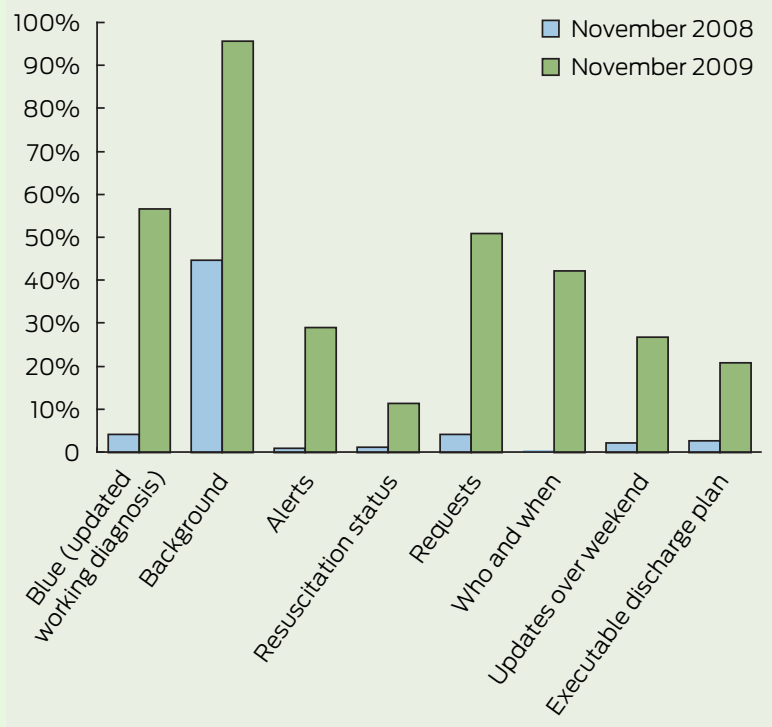




\section{Proportion of general medical inpatients discharged on weekends from Geelong Hospital before and after implementation of the Blue BARRWUE electronic handover system}

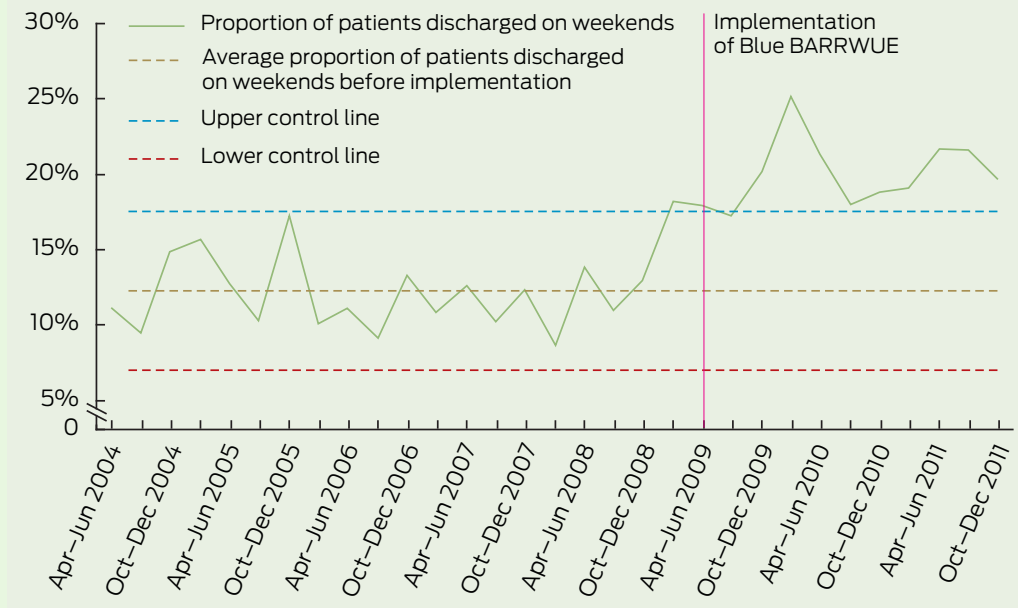

Statistical process control charts were also created for all audits except for the audit of the content of handover notes. These charts included at least 20 data points preceding implementation through to 31 December 2011. Average and upper and lower control lines two standard deviations from the average were calculated using baseline data prior to the implementation of the new handover system. Crossing a control line or eight consecutive data points either above or below the average was considered statistically significant.

\section{Ethics approval}

The research was approved by the Barwon Health Research and Ethics Advisory Committee.

\section{Results}

There were 2034 general medical inpatients in the 12 months before implementation who met our audit inclusion criteria, and 1731 in the 12 months after implementation.

\section{Handover note}

In the 12 months before implementation, 976 patients $(47.98 \%)$ had a handover note in BOSSnet, versus 1646 patients $(95.09 \%)$ in the 12 months after implementation $(P<0.001$; rate ratio $[R R], 20.75$ for presence of a handover note after implementation; 95\% CI, 16.33-26.44; Box 2).

\section{Handover content}

The presence of all components of the handover in November 2009 (6 months after implementation) increased in comparison with that in November 2008 ( $P<0.001$ for all components; Box 3$)$.

\section{Weekend discharges}

In the 12 months before implementation, 289 patients $(14.21 \%)$ were discharged over weekends, versus 353 $(20.39 \%)$ in the 12 months after implementation $(P<0.001 ; R R, 1.44$ for weekend discharge after implementation; 95\% CI, 1.25-1.65; Box 4).

\section{Weekend MET and code blue calls}

There were 152 general medical patients for whom MET calls were made in the 12 months before implementation, (7.47\%), versus 95 general medical patients $(5.49 \%)$ for whom MET calls were made in the 12 months after implementation $(P=$ 0.01; RR, 0.73 for MET calls after implementation; 95\% CI, 0.57-0.94; Box 5).

There were 264/7771 non-general medical patients $(3.40 \%)$ for whom MET calls were made for in the 12 months before implementation, versus 322/8263 non-general medical patients (3.90\%) for whom MET calls were made in the 12 months after implementation $(P=0.09$; RR, 1.15 for MET calls after implementation; 95\% CI, 0.98-1.35). There was no significant change in the small number of code blue calls.

\section{Proportion of general medical patients for whom MET calls were made on weekends, and trends before and after implementation of the Blue BARRWUE electronic handover system* \\ - Proportion of patients with MET call made \\ - Trend before implementation \\ Trend after implementation}

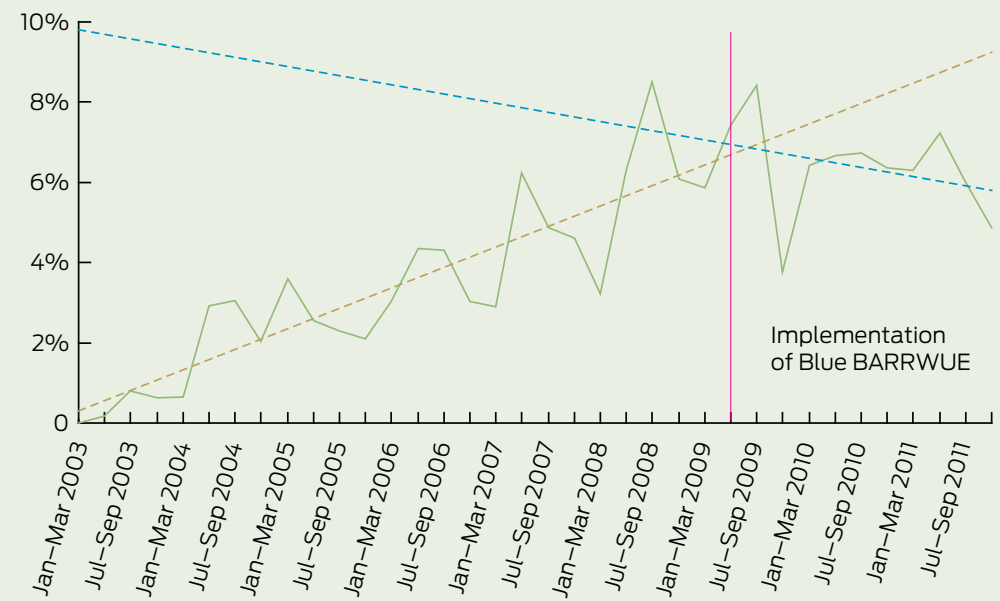

* Steady increase in MET calls before implementation and trend in MET calls after implementation did not reach significance by statistical process control analysis. 


\section{Discussion}

Our brainstorming session led to a standardised but flexible format for written handover that was practical and easy to use and resulted in immediate and sustained uptake. Involving clinicians in the development of handover systems has been reported to be useful and certainly allowed us to identify components that we otherwise would have missed. ${ }^{16}$ As a result, the components of the handover have been remarkably stable - we have not identified any new components that we need to include nor found that any of the existing ones are not relevant - since the implementation of the Blue BARRWUE system.

The presence of handover notes in the clinical handover system now approaches $100 \%$, and content has improved. Recording of the individual components has not been universal, but the system is designed to be flexible and we do not expect all handovers to include all components on any weekend. The proportion of handover notes with resuscitation status present has been the lowest, but it must be remembered that this information cannot be added at the time of the handover unless it has already been discussed with the patient. We do find that resuscitation status is recorded for patients for whom it is most relevant.

Weekend discharges have increased since implementation. Weekend MET calls fell in the 12 months after implementation in general medicine but not in the rest of the hospital (by chisquared analysis). Statistical process control analysis of MET calls was made difficult by the fact that the baseline had been steadily and progressively increasing up to the point of our intervention. Thus, realistically, only about 1 year of data before implementation was worth using as a comparison, but breaking this down into 20 preceding data points made the numbers in each data point very small. The ongoing data and trend line to the end of 2011 strongly suggest that this decline is a real phenomenon. We are not aware of any other changes during this time that might explain the decline.

We believe that the new handover system has had a high sustained uptake as a result of clinical championing by the Head of General Medicine and because of the high value it provides to junior doctors in their daily work. ${ }^{17}$ The handover is quick and easy to enter into the system, it is flexible and highly visible, and junior doctors use it as an aide-mémoire on their daily rounds and when referring patients, writing discharge summaries and covering difficult shifts. Junior doctors have said that covering without it is like "flying blind".

One limitation of this study is that it is historically controlled, and other factors might have affected weekend discharges and MET calls during the follow-up period. Indeed, there is a suggestion that weekend discharges increased just before the new handover system commenced. However, it is unlikely that such increases in weekend discharges would be sustainable without a reliable handover system to underpin them. Some of the improvements may not relate directly to the Blue BARRWUE handover system itself, but to the Friday reminders to complete the handover and combined views in BOSSnet for all patients in the general medical units. The Friday reminders and combined views were developed at the same time as Blue BARRWUE for the purpose of weekend cover.

While the components of the Blue BARRWUE handover system should be broadly generalisable, the results of our implementation probably hinged, at least in part, on having a committed clinical champion and excellent information technology infrastructure. To date, the results of implementation of the program in other parts of Barwon Health have been slower and less reliable without a clinical champion in those areas.

Finally, the "blue" in Blue BARRWUE relates to its colour in BOSSnet specifically, and the acronym may need to be modified for use with other clinical information systems.

Acknowledgements: We would like to thank all the medical registrars and interns who helped create and have used the BlueBARRWUE handover system, Margaret
Henry and Deb Friedman for statistical assistance and Ross Arblaster for creating the queries and reports for data analysis.

Competing interests: No relevant disclosures.

Received 12 Aug 2011, accepted 26 Jul 2012.

1 Van Eaton EG, Horvath KD, Lober WB, et al. A randomized, controlled trial evaluating the impact of a computerized rounding and sign-out system on continuity of care and resident work hours. J Am Coll Surg 2005; 200: 538-545.

2 Petersen LA, Orav EJ, Teich JM, et al. Using a computerized sign-out program to improve continuity of inpatient care and prevent adverse events. Jt Comm J Qual Improv 1998; 24: 77-87.

3 Bhabra G, Mackeith S, Monteiro P, Pothier DD. An experimental comparison of handover methods. Ann R Coll Surg Engl 2007; 89: 298-300.

4 Porteous JM, Stewart-Wynne EG, Connolly M, Crommelin PF. ISoBAR - a concept and handover checklist: the National Clinical Handover Initiative. Med J Aust 2009; 190 (11 Suppl): S152-S156.

5 Haig KM, Sutton S, Whittington J. SBAR: a shared mental model for improving communication between clinicians. Jt Comm J Qual Patient Saf 2006; 32: 167-175.

6 Finnigan MA, Marshall SD, Flanagan BT. ISBAR fo clear communication: one hospital's experience spreading the message. Aust Health Rev 2010; 34 400-404.

7 Marshall S, Harrison J, Flanagan B. The teaching of a structured tool improves the clarity and content of interprofessional clinical communication. Qual Saf Health Care 2009; 18: 137-140.

8 Cheah LP, Amott DH, Pollard J, Watters DA. Electronic medical handover: towards safer medical care. Med J Aust 2005; 183: 369-372.

9 Hodgetts TJ, Kenward G, Vlachonikolis IG, et al. The identification of risk factors for cardiac arrest and formulation of activation criteria to alert a medical emergency team. Resuscitation 2002; 54: 125-131.

10 Buist M, Bernard S, Nguyen TV, et al. Association between clinically abnormal observations and subsequent in-hospital mortality: a prospective study. Resuscitation 2004; 62: 137-141.

11 Cretikos M, Chen J, Hillman K, et al; MERIT study investigators. The objective medical emergency team activation criteria: a case-control study. Resuscitation 2007; 73: 62-72.

12 Jones D, George C, Hart GK, et al. Introduction of medical emergency teams in Australia and New Zealand: a multi-centre study. Crit Care 2008; 12: R46.

13 Chen J, Bellomo R, Flabouris A, et al. The relationship between early emergency team calls and serious adverse events. Crit Care Med 2009; 37: 148-153.

14 DeVita MA, Braithwaite RS, Mahidhara R, et al; Medical Emergency Response Improvement Team. Use of medical emergency team responses to reduce hospital cardiopulmonary arrests. Qual Saf Health Care 2004; 13: 251-254.

15 Jones D, Bellomo R, DeVita MA. Effectiveness of the Medical Emergency Team: the importance of dose. Crit Care 2009; 13: 313.

16 Wong MC, Turner P, Yee KC. Involving clinicians in the development of an electronic clinical handover system - thinking systems not just technology. Stud Health Technol Inform 2008; 136: 490-495.

17 Soo S, Berta W, Baker GR. Role of champions in the implementation of patient safety practice change. Healthc Q 2009; 12 Spec No Patient: 123-128. 